\title{
Controlled release nanoparticle-embedded coatings reduce the tissue reaction to neuroprostheses
}

\author{
André Mercanzini a,b,* Sai T. Reddy ${ }^{\mathrm{a}, \mathrm{c}}$, Diana Velluto ${ }^{\mathrm{a}, \mathrm{c}}$, Philippe Colin ${ }^{\mathrm{a}, \mathrm{d}}$, Anne Maillard ${ }^{\mathrm{a}, \mathrm{d}}$, \\ Jean-Charles Bensadoun ${ }^{\mathrm{a}, \mathrm{d}}$, Jeffrey A. Hubbell ${ }^{\mathrm{a}, \mathrm{c}}$, Philippe Renaud ${ }^{\mathrm{a}, \mathrm{b}}$ \\ a Ecole Polytechnique Fédérale de Lausanne, Switzerland \\ b Microsystems Laboratory, STI-LMIS4, station 17, EPFL, 1015 Lausanne, Switzerland \\ c Laboratory for Regenerative Medicine and Pharmacobiology, SV-IBI, station 15, 1015 Lausanne, Switzerland \\ d Neurodegenerative Diseases Laboratory, SV-BMI, station 15, 1015 Lausanne, Switzerland
}

\section{A R T I C L E I N F O}

\section{Article history:}

Received 10 January 2010

Accepted 27 April 2010

Available online 4 May 2010

\section{Keywords:}

Microfabrication

Nanoparticles

Neuroprosthesis

\begin{abstract}
A B S T R A C T
Controlled release coatings were developed for neuroprostheses with the aim of combating the tissue reaction following implantation in the brain. The coatings consist of poly(propylene sulfide) drug-eluting nanoparticles embedded in a poly(ethylene oxide) matrix. The nanoparticles are loaded with dexamethasone, an anti-inflammatory drug known to have an effect on the cells activated during the damage caused by implantation. The nanoparticles are not affected by the coating process and the drug remains bioactive after it is released. The coating was applied to microfabricated cortical neuroprostheses consisting of platinum and polyimide. Coated drug-eluting devices were implanted in the cortex of rats. After implantation the matrix dissolves, exposing the electrode surfaces, while the nanoparticles remain in the vicinity of the tissueimplant interface. Using electrical impedance spectroscopy and comparative histology, a long-term decrease in the tissue response in comparison to control devices was observed. These coatings can therefore be used to increase the reliability and long-term efficacy of neuroprostheses.
\end{abstract}

(C) 2010 Elsevier B.V. All rights reserved.

\section{Introduction}

Microelectrodes implanted in nervous tissue can be used to stimulate or record neural activity and may one day reproduce neural functions lost to trauma or disease [1]. A limiting factor with microelectrodes chronically implanted in the brain is their loss of electrical contact with neural tissue due to the post-implantation inflammatory reaction, gliosis, and fibrosis [2-4]. Glial cells rapidly migrate to the implantation site surrounding the device, thus physically separating the microelectrode sites from the neurons they are meant to be recording from or stimulating. The efficiency of the implanted device decreases steadily with time. After approximately two to three weeks, the inflammatory response has reached its peak, electrical impedance measurements have stabilized at their maximum [5], and the number of chronically recorded single units has reached its minimum [6]. If microelectrode arrays are to be effective in neural stimulation and recording, the tissue response must be reduced or prevented in order to maintain stable microelectrodetissue contact and neuroprostheses functionality.

Several approaches to limiting this tissue reaction have been reported, including surface modification to prevent cell adhesion [7],

\footnotetext{
* Corresponding author. Tel.: +41 77252 2402; fax: +41 216935950. E-mail address: andre.mercanzini@epfl.ch (A. Mercanzini).
}

localized release of alpha-melanocyte stimulating hormone ( $\alpha$-MSH) [8] or nerve growth factor $\beta$ [9], and dexamethasone injection [10]. The approach presented here involves coating microelectrode arrays with bio-resorbable drug-eluting material. We hypothesized that highly localized delivery of an anti-inflammatory drug around the implantation site would reduce the tissue response to implantation and improve recording and stimulation characteristics. The goal of this work was to determine the efficacy of these drug-eluting coatings to combat the inflammatory response to implantation.

Novel nanoparticle-embedded coatings were designed and evaluated for the controlled release of dexamethasone, which has been shown to be effective in reducing the brain response to implantable devices $[10,11]$. Its release from poly(lactic-co-glycolic acid) (PLGA) nanoparticles was shown by Kim and Martin [12] using rigid silicon probes, however in this experiment the neuroprostheses were polymeric, resulting in less post-implantation injury and eventually a more straightforward translation into clinical use.

Dexamethasone was loaded into poly(propylene sulfide) (PPS) nanoparticles which were then incorporated into poly(ethylene oxide) (PEO), a bio-resorbable polymer, and applied as a coating to the neuroprosthesis. The PEO is meant to dissolve soon after implantation, thereby releasing the drug-loaded nanoparticles and exposing the electrode sites. The nanoparticles are large enough to not diffuse from the surface of the implanted device and can maintain sustained release at the implantation site. The nanoparticles were 
characterized before and after the coating process, to ensure that they were not damaged using scanning electron microscopy (SEM) and in vitro release rates were obtained. In vivo efficacy in the brain was evaluated using two different methods in the rat model. The first was by measuring the electrical characteristics of the tissue reaction using electrical impedance spectroscopy. The second was with immunofluorescence which gives qualitative insight into the cell morphology around the device.

We demonstrate with this study that the highly localized release of dexamethasone around the neuroprosthesis indeed reduces the longterm tissue reaction. In stimulation applications of neuroprostheses, a reduction in the tissue reaction will enable more efficient charge transfer during neural stimulation, reducing the current required and preventing toxic electrochemical reactions at the metal microelectrode surface [13]. In recording applications of neuroprostheses, the signal-to-noise ratio may be improved if the degree of the tissue reaction is reduced $[14,15]$. These are important factors for the continued use of microelectrode based neuroprostheses in research, and may accelerate the translation of this technology into clinic applications.

\section{Materials and methods}

\subsection{Microelectrode array fabrication}

Several materials and processes to make neuroprostheses using thin film technology have been shown [16-18]. The devices presented here are based on polyimide-platinum microfabrication, for which the full fabrication method has been previously published $[19,20]$. These devices have also been demonstrated in vivo for both acute and chronic measurements [20,21]. Fig. 1A) demonstrates the device used in these experiments, while Fig. 1B) shows a close-up of the tip dimensions. The tip was composed of four microelectrodes of $50 \mu \mathrm{m}$ diameter with spacing of $150 \mu \mathrm{m}$ or $300 \mu \mathrm{m}$. The device thickness was $60 \mu \mathrm{m}$.

Each device consisted of two probes, one for each hemisphere of the brain, totaling 8 microelectrodes per animal. One probe was coated with the drug-eluting material, while the other probe was coated with the non-drug-loaded coating and served as the control. The stiffness of flexible devices may be an issue during implantation in deeper areas of the brain. The devices are flexible, but stiff enough [22] to penetrate the cortical tissue by more than $1 \mathrm{~mm}$.
A

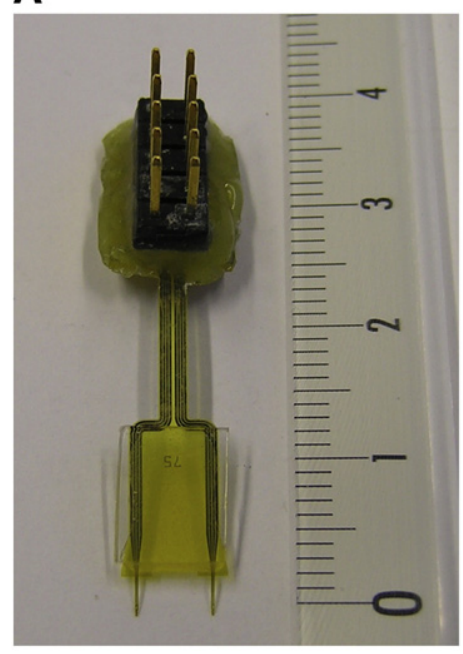

B

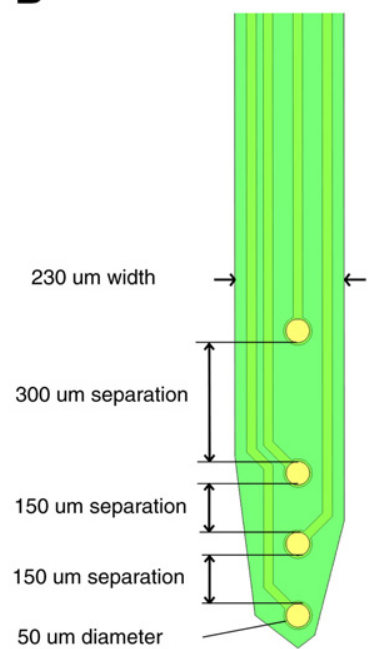

Fig. 1. A) The implantable device demonstrating the left and right hemisphere probes, and the electrical connector which remains extracorporeal. The scale is in centimeters. B) The design of the probe tip demonstrating microelectrode sizes and spacing. Adapted from Ref. [5].
Separate microelectrode arrays for histological evaluation were fabricated using the same process described above, but with slightly different dimensions such that the short histology microelectrode array completely surpassed the dura mater to prevent it from adhering to the inside of the skull.

\subsection{Nanoparticle-PEO coating synthesis}

\subsubsection{Nanoparticle preparation}

We follow the procedure for synthesizing nanoparticles described elsewhere $[23,24]$. For further applications of the nanoparticles, the reader is directed to Reddy et al. [25,26]. All solvents and reagents were purchased from Sigma-Aldrich (Buchs, Switzerland). Doubledistilled and purified water was deoxygenated by bubbling nitrogen gas in a slight vacuum environment and stirring at $1000 \mathrm{rpm}$ for $1 \mathrm{~h}$. While flooding the beaker with nitrogen gas, $35 \mathrm{mg}$ of Pluronic F$127 \circledR$ was added and stirred at $1000 \mathrm{rpm}$ for $1 \mathrm{~h}$ until completely dissolved. $400 \mu \mathrm{L}$ of propylene sulfide was added to the solution continuously under nitrogen purge and was allowed to mix for 5 min before adding the initiator solution. The initiator consists of unprotected pentaerythritol tetrathioester and was prepared as described elsewhere [27]. Sodium methoxide in methanol was used to activate the $\mathrm{SH}$ end groups (i.e. deprotect) of the pentaerythritol tetrathioester and initiate polymerization of the propylene sulfide monomer. $200 \mu \mathrm{L}$ of $0.5 \mathrm{M}$ sodium methoxide was added to the pentaerythritol tetrathioester in a nitrogen gas environment. The initiator solution was then added to the monomer and Pluronic ${ }^{\circledR}$ surfactant solution and stirred for $3 \mathrm{~min}$ in a constant nitrogen environment. $60 \mu \mathrm{L}$ of the organic base 1,8-diazabicyclo [5.4.0] undec7 -ene was added to the solution. This reagent is highly hydrophobic and is believed to improve particle formation. The solution was allowed to stir overnight and upon exposure to air the PPS becomes oxidized, resulting in disulfide formation, and thus a crosslinked PPS core. The nanoparticle solution was then purified by dialysis in ultrapure Millipore water with a $100 \mathrm{kDa}$ dialysis membrane for 3 days with 2 buffer changes per day, ensuring that the solution is void of unreacted Pluronic ${ }^{\circ}$, PPS and reagents. Once formed, nanoparticles can be kept in solution for several months at $4{ }^{\circ} \mathrm{C}$ without apparent degradation.

\subsubsection{Dexamethasone loading}

To deliver therapeutic drug amounts, the nanoparticles must be efficiently loaded to ensure high drug concentration and this was achieved by a cosolvent-evaporation in water technique [28]. $5 \mathrm{mg}$ of dexamethasone was added to $400 \mu \mathrm{L}$ of methanol and stirred until dissolved. $1 \mathrm{~mL}$ of the nanoparticle solution described above was added to the dexamethasone-methanol solution and the mixture was stirred for $8 \mathrm{~h}$ covered only by a particle free tissue to allow the solvent to evaporate. During the cosolvent (methanol) evaporation, the hydrophobic dexamethasone molecule will permeate the Pluronic ${ }^{\circledR}$ corona of the nanoparticles and remain in the hydrophobic PPS core. The solution was then purified by centrifugation at $11 \mathrm{krpm}$ $(6800 \mathrm{~g})$ for $10 \mathrm{~min}$. After centrifugation, a precipitate of unloaded dexamethasone was visible at the bottom of the vial. The weight of this remaining dexamethasone represents the unloaded drug. The supernatant consists of the dexamethasone-loaded nanoparticles and was removed for sizing experiments and for coating preparation. The nanoparticle solution was sterile filtered through a $0.22 \mu \mathrm{m}$ filter.

\subsubsection{Nanoparticle incorporation with PEO}

The nanoparticle-dexamethasone solution was suspended in a PEO matrix, and then applied to the neural probes using a dip and reflow method. This forms uniform films around the probe.

The PEO solution was prepared by dissolving $200 \mathrm{mg}$ of $1,000,000 \mathrm{~g} / \mathrm{mol}$ molecular weight PEO (Sigma-Aldrich, Switzerland) in $8 \mathrm{~mL}$ of purified water. A $1 \mathrm{~mL}$ suspension of dexamethasone- 
loaded nanoparticles in DI water was slowly added to $1 \mathrm{~mL}$ of PEO solution and stirred in order to disperse the nanoparticles. A neural probe was inserted into the viscous solution and quickly pulled out. The PEO-nanoparticle suspension was allowed to air dry, forming a non-uniform layer around the neural probe. The coated probe was then placed in an oven at $60{ }^{\circ} \mathrm{C}$ for $2 \mathrm{~min}$ and the PEO-nanoparticle suspension was allowed to reflow around the probe forming a smooth and uniform coating around the device. The coated neural probe can then be stored at $5{ }^{\circ} \mathrm{C}$ for several hours before implantation. Control probes were prepared in precisely the same manner, except the nanoparticles used in the preparation had not been loaded with dexamethasone.

\subsubsection{Nanoparticle characterization}

The produced nanoparticles were characterized for their size, robustness after the coating process, release rate, and retained bioactivity after release. A dynamic light scattering instrument (Zetasizer Nano Series, Malvern Instruments Ltd, UK) was used to measure a sample of the solution to determine the average nanoparticle diameter before loading of dexamethasone.

Nanoparticles were imaged in order to confirm correct nanoparticle formation and retained structural integrity after embedment within the PEO matrix and subsequent re-hydration. Nanoparticles were imaged using a modern electron microscopy technique which permits study of materials in atmospheric and aqueous solutions within a scanning electron microscope (SEM). This technique differs from so-called "environmental SEM" because it can be performed in a conventional SEM using QX-102 WETSEM ${ }^{\circledR}$ capsules purchased from Quantomix Corporation (Rehovot, Israel). Two nanoparticle solution samples were prepared and placed in two such capsules. The first sample consisted of nanoparticles after dexamethasone loading. The second sample consisted of loaded nanoparticles that had been embedded in the PEO matrix, followed by dehydration of the PEO matrix in the same manner used during coating, and finally rehydrated. The second sample closely resembles the coating process and evaluates the robustness of the nanoparticles.

The release rate of the nanoparticles was determined using an in vitro dialysis procedure which helped model in vivo conditions. A $1 \mathrm{~mL}$ sample of loaded nanoparticle solution was placed in a cellulose dialysis membrane with a nominal molecular weight cutoff of $12 \mathrm{kDa}$ (Cole Parmer, IL, USA). The membrane was placed in a $1 \mathrm{~L}$ beaker of purified water for 10 days. Daily samples of $100 \mu \mathrm{L}$ were removed from the membrane, and immediately freeze dried. The daily samples demonstrate a decreasing concentration of dexamethasone, as it slowly diffuses from the nanoparticles and out of the dialysis membrane.

The freeze dried samples representing daily dexamethasone concentration were then dissolved in $100 \mu \mathrm{L}$ of tetrahydrofuran (THF) (Sigma-Aldrich, Switzerland). Gel permeation chromatography was used to determine the exact concentration of bioactive dexamethasone in these samples in comparison to standard concentration measurements.

Samples (in THF) were injected into three serial Waters Styragel HR columns (Waters Corp, Delaware). A $1 \mathrm{~mL} / \mathrm{min}$ THF mobile phase set at $40{ }^{\circ} \mathrm{C}$ allowed the detection of the polymer using a differential refractometer detector (model 410, Waters) or a UV detector (photodiode array detector, Waters Corp. model 996).

\subsection{Implantation}

Animal care and experimental procedures were performed in accordance with the Swiss legislation on animal protection and experimentation. Six adult female RCS rats were anesthetized with xylasine (Narcoxyl ${ }^{\circledR} 2$ at $10 \mathrm{mg} / \mathrm{kg}$ ) and ketamine (Ketaminol ${ }^{\circledR} 10$ at $75 \mathrm{mg} / \mathrm{kg}$ ). Subjects were implanted using a stereotaxic frame in the primary motor cortex. Four subjects received devices for impedance measurements, and two subjects received devices for histology. Each subject had one hemisphere implanted with a drug-eluting probe, and the opposite hemisphere with a control probe. The four subjects used for impedance measurements had four microelectrodes per hemisphere, resulting in 32 microelectrode sites that were measured during the experiment.

Stereotactic coordinates were set to +1.5 anterior, $+2.5 \mathrm{~mm}$ and $-2.5 \mathrm{~mm}$ lateral from bregma, toothbar was $-3.3 \mathrm{~mm}$ [29]. Fig. 2 demonstrates a coronal image of the device placement. An incision was made to expose the skull and six $2 \mathrm{~mm}$ diameter craniotomies were performed remove the skull and pierce the dura mater. Once the device was implanted it was stabilized using dental acrylic. The skin was sutured and animals recovered in a warm environment.

Two of the craniotomies were used as device implantation sites and the remaining four were used for self-tapping stainless steel screws, which served to stabilize the implant using dental acrylic after implantation. The two rear screws also served as the signal ground and must be in contact with the cortex. Animals were kept for a total of 46 days.

\subsection{In vivo impedance measurements}

Experimental measurements of tissue reaction properties were obtained for each animal subject during the study. Subjects were measured while temporarily anesthetized daily for the first two weeks and every three days until animal sacrifice. Electrical impedance spectroscopy measurements were performed with an Agilent 4294A impedance analyzer connected to a routing circuit which directs the impedance measurement signal to each microelectrode site. The impedance of each individual microelectrode was recorded using proprietary MATLAB ${ }^{\circ}$ software. A frequency sweep from $100 \mathrm{~Hz}$ to $1 \mathrm{MHz}$ was performed at $100 \mathrm{mV}$ amplitude, thereby sufficiently covering the bandwidth of electrophysiological interest and identifying important material properties.

The experiments and model described by Mercanzini et al. [5] demonstrate how the exact resistance of the tissue reaction can be determined using the technique of Peak Resistance Frequency analysis. This method is more powerful than conventional impedance measurements at $1 \mathrm{kHz}$ because it isolates and identifies the resistance of the tissue surrounding the electrode. The reader is referred to this reference for a more detailed explanation of the Peak Resistance Frequency.

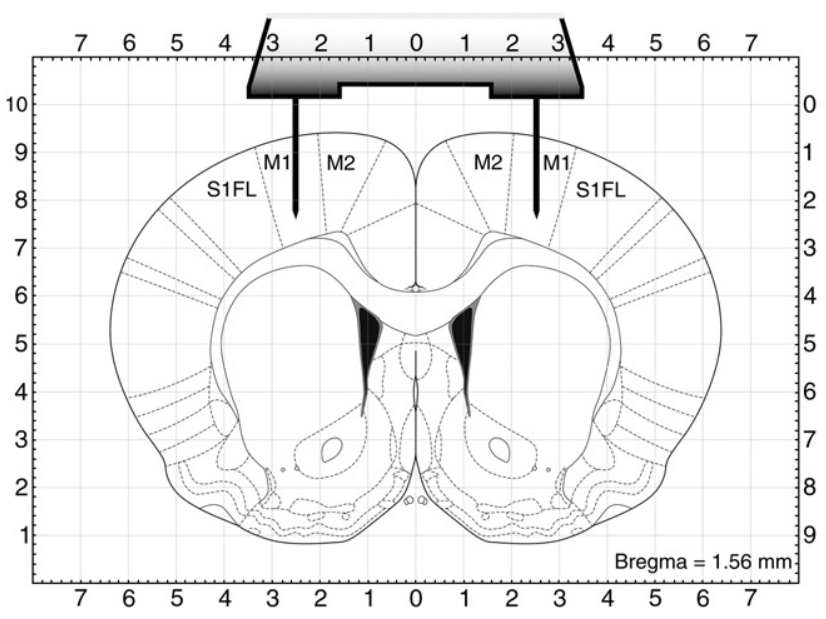

Fig. 2. A coronal slice demonstrating microelectrode array placement in the primary motor cortex (M1).

Adapted from Ref. [29]. 


\subsection{Histology}

Separate implants were made for histology that did not have electrical contacts leading outside of the animal in order to facilitate microtome sections of the brain with the microelectrode array present. This does not permit a direct quantitative correlation between histology and electrophysiological data, but further development on histological techniques may permit such an experiment.

At 1 and 3 week time points, rat brains were transcardially perfused with a $4 \%$ paraformaldehyde-containing saline solution. A paraffin microtome (Microm HM325, Microm Microtech, France) was used to slice the tissue with electrodes in place in $25 \mu \mathrm{m}$ slices. Slices were de-parafinized by heating to $60{ }^{\circ} \mathrm{C}$ for $20 \mathrm{~min}$ and immediately rinsing in toluene twice for $5 \mathrm{~min}$ each. The slices were then rinsed with ethyl alcohol at concentrations of $100 \%, 95 \%, 80 \%$ and $65 \%$ for 5 min at each concentration then washed in DI water. The slices were then incubated in $10 \mathrm{mM}$ citric acid ( $\mathrm{pH} \mathrm{6)}$ at $90{ }^{\circ} \mathrm{C}$ for $20 \mathrm{~min}$ and allowed to cool (antigen retrieval) and then washed in PBS twice for $5 \mathrm{~min}$ each. Slices were incubated at room temperature for one hr in a solution consisting of $1 \% \mathrm{BSA}, 2 \%$ donkey serum and $0.1 \%$ Triton-X in PBS. Slices were then incubated for $12 \mathrm{~h}$ at $4{ }^{\circ} \mathrm{C}$ with the primary antibodies: $0.5 \mu \mathrm{L} / \mathrm{mL}$ rabbit anti-Iba1 (Wako, IG Instrument, Zurich, Switzerland) was the microglia marker and $1.25 \mu \mathrm{L} / \mathrm{mL}$ mouse antiglial fibrillary acidic protein (GFAP; Dako, Sigma-Aldrich, Buchs, Switzerland) was the astrocyte marker. After washing three times in PBS for 5 min each, the slides were incubated at room temperature in the secondary antibodies: Cy3 donkey anti-mouse $1.7 \mu \mathrm{L} / \mathrm{mL}$ and Alexa 488 donkey anti-rabbit $1 \mu \mathrm{L} / \mathrm{mL}$. The slides were washed three times in PBS for 5 min each and incubated in 4',6-diamidino-2-

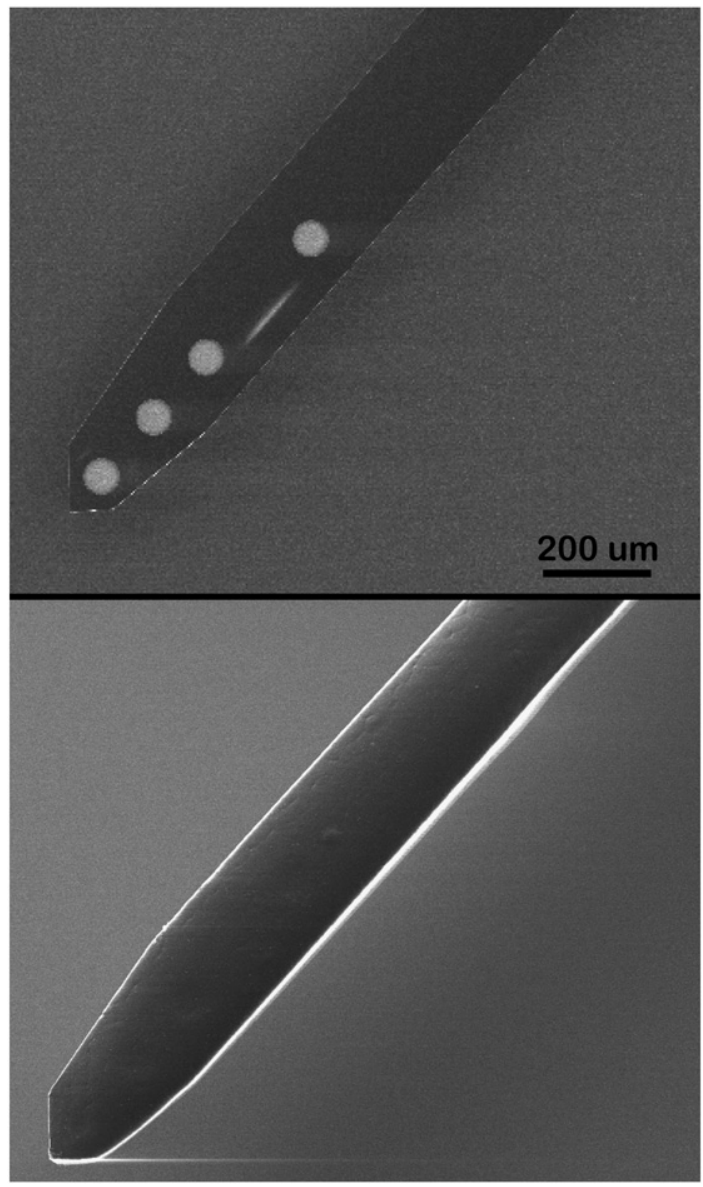

Fig. 3. A neural probe tip before coating (top image) and after the coating process (bottom image). The scale bar applies to both images. Coating thickness was also determined using electron microscopy to be $30 \mu \mathrm{m}$. Dimensions are given in Fig. 1 .

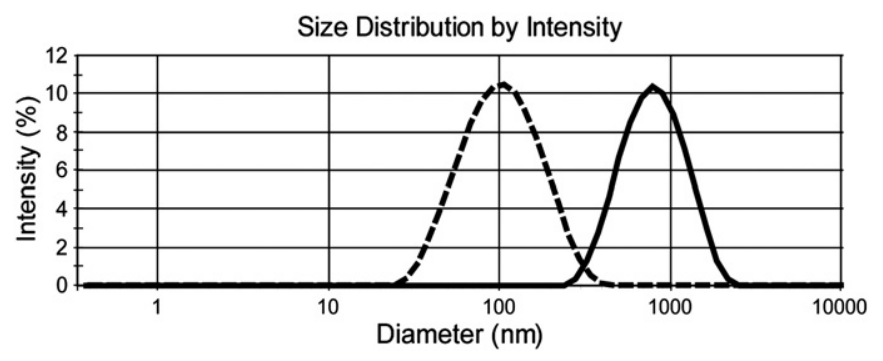

Fig. 4. Median nanoparticle size distribution as determined using dynamic light scattering (note that $x$-axis is a log scale). Before embedding in the PEO matrix the nanoparticles demonstrate a median diameter of $100 \mathrm{~nm}$ (dashed line). After embedding in the PEO matrix and subsequent, dehydration, and re-hydration, the nanoparticles demonstrate a median diameter of $800 \mathrm{~nm}$ (solid line) indicative of aggregation.

phenylindole (DAPI), a nuclei marker for all cells, at a concentration of $1 \mu \mathrm{L} / \mathrm{mL}$ for $15 \mathrm{~min}$. The slides were washed twice for 5 min each in PBS and bonded with cover slips using water-based glue.

\section{Results}

\subsection{Nanoparticle-PEO coating synthesis}

Fig. 3 demonstrates a neural probe tip before and after coating. The average dimensions of the coating are $230 \mu \mathrm{m}$ wide, $30 \mu \mathrm{m}$ thick, and approximately $1000 \mu \mathrm{m}$ in length. The coating is present on both sides of the implant giving a final coating volume of $\sim 15 \mathrm{~nL}$.

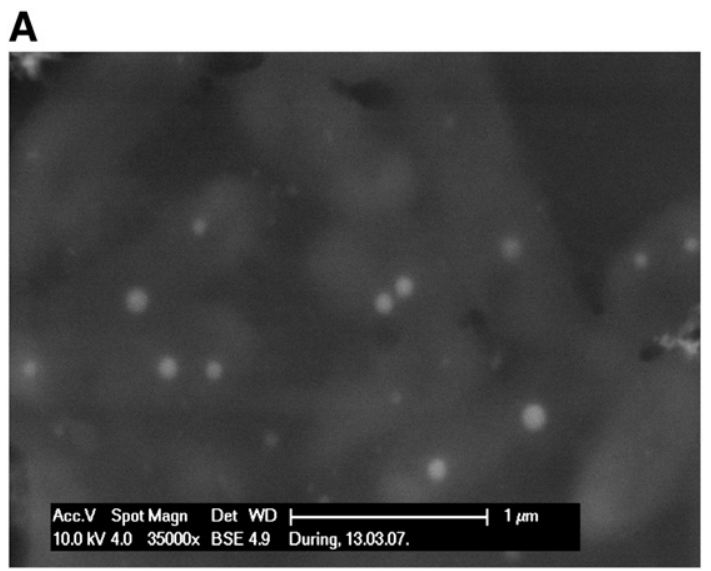

B

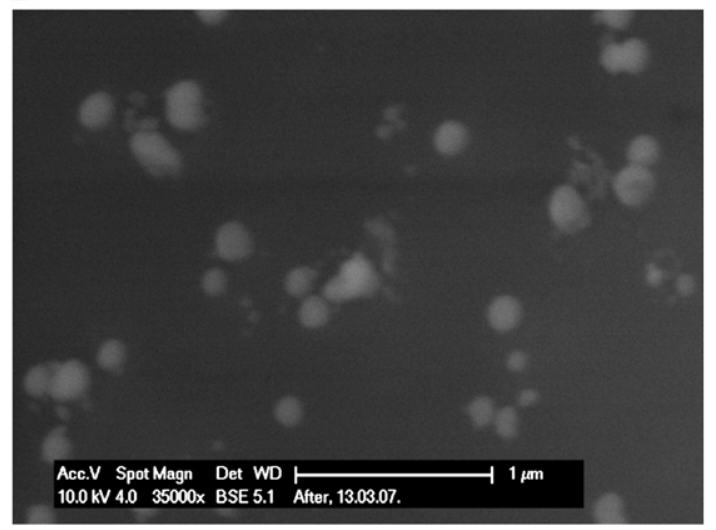

Fig. 5. WETSEM ${ }^{\circledR}$ images of PPS nanoparticles. A) The loaded nanoparticles appear as white colored spheres, demonstrating diameters in range of $80-100 \mathrm{~nm}$ as expected from the DLS measurements. B) The nanoparticles following the steps of embedded in the PEO matrix, dehydration, and re-hydration. The nanoparticles became significantly larger but retained their dispersed structure. 


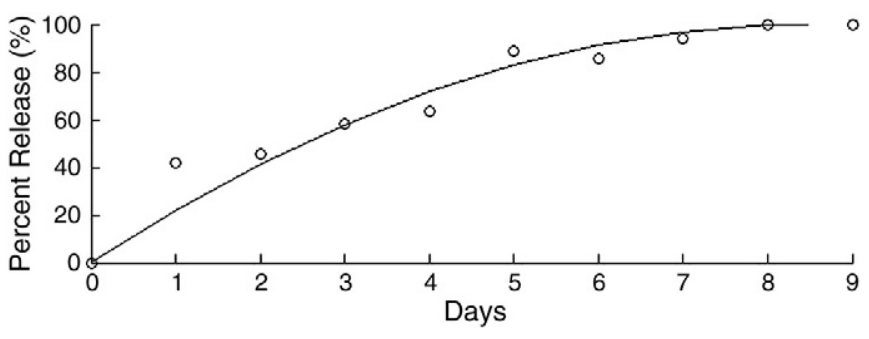

Fig. 6. Percent release rate of dexamethasone-loaded nanoparticles. Complete drug release was achieved after 9 days.

The nanoparticle median diameter as measured with DLS is depicted in Fig. 4 before and after integration within the PEO matrix. An average diameter of $100 \mathrm{~nm}$ is measured for the nanoparticles before integration. After integration and subsequent dissolution, the nanoparticles had an apparent increase in diameter to $800 \mathrm{~nm}$ on average. This does not suggest an increase in the size of the PPS core, but may be due to long PEO chains wrapping around its surface, in addition to possible aggregation of several particles. However, this does not affect the capability of the particles to retain or release the loaded hydrophobic molecule as demonstrated by the release experiments.

Imaging the nanoparticles using electron microscopy demonstrated that they retained a spherical shape after the coating and release process. Fig. 5A) demonstrates the nanoparticles immediately after loading with dexamethasone. Fig. 5B) demonstrates the nanoparticles after re-hydration and release from the PEO matrix. They have increased noticeably in size, which is in agreement with DLS measurements.

The dexamethasone was loaded into the nanoparticles with a maximum encapsulation efficiency of $15 \% \mathrm{w} / \mathrm{w}$. The release rate over 9 days is depicted in Fig. 6. Data was fitted to a quadratic curve to guide the eye but is not meant to model release rate. A rapid release up to $40 \%$ of the dexamethasone content was seen in the first day, and then a steady release continued until dexamethasone traces are no longer detected.

The dosage of drug surrounding the implant can be calculated by considering the concentration of dexamethasone in the nanoparticle solution and the geometry of the coating. The nanoparticledexamethasone solution was formulated with $5 \mathrm{mg} / \mathrm{mL}$ of which $15 \%$ was retained in the nanoparticles. Therefore the concentration of dexamethasone in the nanoparticle solution was $750 \mu \mathrm{g} / \mathrm{mL}$. This was mixed into a PEO-water hydrogel at a 1:1 ratio, further diluting the dexamethasone to $375 \mu \mathrm{g} / \mathrm{mL}$. However, during the coating process most of the water will evaporate from the PEO-nanoparticle mixture. Conservatively assuming that the final coating retains $20 \%$ moisture,
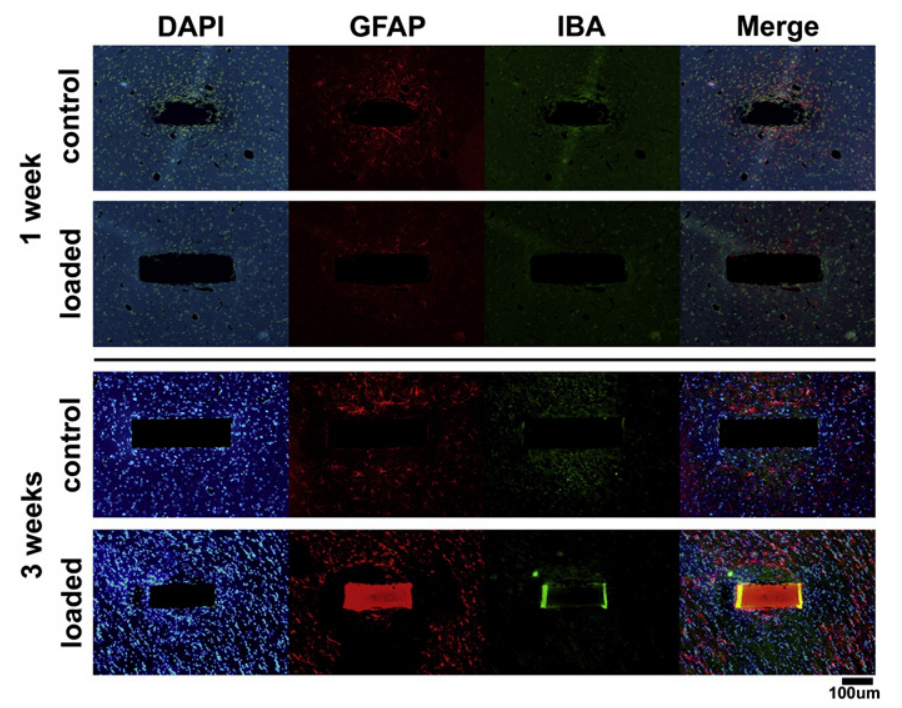

Fig. 8. Histological results comparing control and drug-loaded nanoparticle devices. DAPI staining is for all cell nuclei, GFAP stains for astrocytes, IBA is for reactive microglia. After 1 week there is a clear difference between the tissue reactions that have formed around the control and drug-eluting probe. After 3 weeks bioimpedance measurements demonstrate a stabilization of the tissue reaction, and this is supported through histology. The reaction for drug-eluting probes is limited compared to the control probes. A lower density of astrocytes is apparent. (Note: The slide which displays the loaded week 3 sample has the remains of an embedded probe within).

the dexamethasone concentration is $300 \mu \mathrm{g} / \mathrm{mL}$ in the coating material. By considering the $15 \mathrm{~nL}$ of coating volume calculated above, we can conclude that a final dosage of $4.5 \mathrm{pg}$ of dexamethasone is present in the immediate vicinity of the microelectrode array.

\subsection{In vivo impedance comparison}

Impedance measurements were performed to determine the effects of the drug release on the electrical properties of the tissue reaction. A total of 32 microelectrode sites were measured (i.e. 16 sites for drug-eluting probes and 16 sites for control). For each day the impedance magnitude at the Peak Resistance Frequency was plotted for the control and drug-eluting sites. Fig. 7 shows the individual daily values isolating the impedance magnitude of the tissue reaction only. The average for each day is demonstrated and a clear trend is apparent. At the conclusion of the 46 day experiment the final average impedance magnitude for drug-eluting probes is $3.15 \mathrm{M} \Omega$, while the average for control probes is $4.17 \mathrm{M} \Omega$. This demonstrates an average

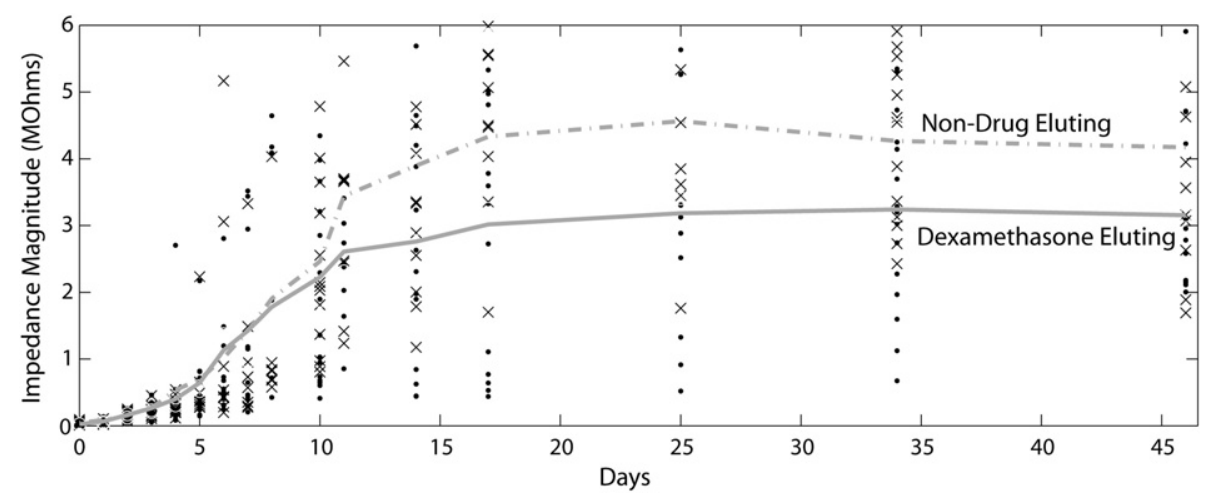

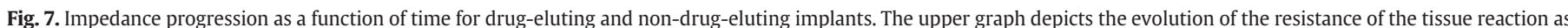

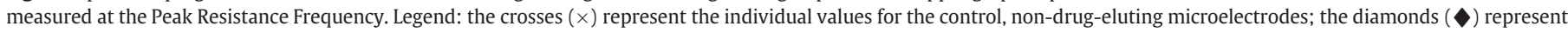

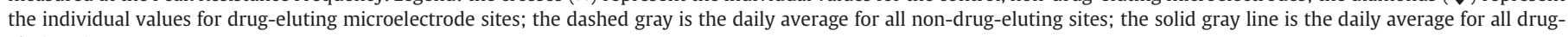
eluting sites. 
$25 \%$ difference in impedance magnitude of the tissue reaction when drug-eluting devices are used.

\subsection{Histological results}

Fig. 8 demonstrates a qualitative histological comparison at two important time points. After one week a substantial increase in the tissue resistance surrounding the microelectrode is observed, as confirmed by impedance measurements. It is apparent that the reaction is limited in comparison to the control device by the first week, and this reduction is maintained by the third week. These results are comparable to the thorough quantitative analysis of Zhong and Bellamkonda [11], however such an analysis is beyond the scope of this paper.

\section{Discussion}

In comparison to loading a biodegradable material with dexamethasone [30], the nanoparticle technique presented here prevents the rapid diffusion of the drug away from the implantation site [31]. After the coating process, nanoparticles are large enough $(800 \mathrm{~nm})$ that they remain close to the implantation site where the highest concentration of the drug is required.

The method of release and degradation of the particles is a combination of oxidation of the PPS core [27] and diffusion. It is not known for the specific target cells (i.e. the astrocytes and microglia) what the drug uptake mechanism in the brain is. However, it is believed that the uptake method is either through passive processes (the drug is released into the vicinity of the cell and uptake happens through diffusion) or the drug-laden nanoparticle is internalized by the microglia by macropinocytosis or endocytosis [23].

In order to encapsulate hydrophobic molecules such as dexamethasone and prevent tissue reaction to the nanoparticles, the materials must have certain core and surface characteristics. The PPS nanoparticles used here have a hydrophobic core and PEGylated, hydrophillic surface $[23,24,27]$. They provide a stable rubbery PPS core in which a hydrophobic drug can be loaded, which may degrade in an oxidative environment. The hydrophilic corona of the nanoparticle will prevent the adhesion of proteins which leads to tissue reaction. These nanoparticles were shown elsewhere to be non-cytotoxic [27].

The polymer coating does not adversely affect the electrical characteristics of the electrode sites as was demonstrated in vitro in a previous study [32]. A coating material was sought that would rehydrate and fully dissolve after implantation, thus fully exposing the electrode sites to the neural tissue. Selection of a high molecular weight of the PEO matrix was important to provide non-instantaneous dissolution after re-hydration during implantation. Low molecular weight PEO or PEG may dissolve during, rather than after, insertion. However, long-term effects of PEG in the brain are still not known and can influence the viability of this technique.

In previous work [12,33], the impedance magnitude at $1 \mathrm{kHz}$ has been used to determine the extent of tissue reactions. However, this method yields less insight than Peak Resistance Frequency analysis because it does not isolate and measure the resistance of the tissue reaction alone. In fact, measuring at this one frequency does not provide any insight into the time-changing activity around the electrode. Only by following the magnitude of the Peak Resistance Frequency for each individual electrode, and determining the average daily impedance, can an accurate representation of the increase in the tissue reaction impedance be possible.

The in vivo experiments demonstrate a decrease by $25 \%$ of impedance magnitude and this result is substantial. For example, it translates into $25 \%$ less current required to stimulate neural tissue. Electrochemical reactions on the surface of the electrode that can occur during stimulation can be avoided if the current required for efficacious stimulation is lower. The decrease in the power required to stimulate has a direct effect on battery life for implantable pulse generators, and longer battery life is an important clinical need. There may also be an increase in the signal-to-noise ratio when recording neural activity. However, in vivo demonstration of improved recording should be the subject of future work.

The impedance measurement methods described in this study can also be used to evaluate and compare different drug-eluting and biofunctional coatings. For example, there have been reports of nanoscale laminin coatings [34] and bioactive coatings [35,36]. These coatings could be applied to the devices presented here and a quantitative comparison of the tissue reaction could be performed. The evaluation and comparison of different dosages or release kinetics can also be performed using this technique. To control release kinetics, coatings may be a combination of several layers with different drug concentrations in order to take advantage of different release kinetics as demonstrated elsewhere [8]. For example, the outer-most layer of the coating may contain the highest concentration of nanoparticles in order to provide a high initial dosage or "burst", while subsequent layers will slowly reduce in drug concentration.

\section{Conclusion}

We have described a method to synthesize nanoparticles with a hydrophobic core made of poly(propylene sulfide) and a hydrophilic corona of poly(ethylene glycol). The nanoparticles were loaded with dexamethasone, a hydrophobic drug, and incorporated it into a high molecular weight poly(ethylene oxide) matrix for use as an antiinflammatory coating on microfabricated polymer neuroprostheses. The nanoparticles protect the drug from degradation, and ensure its controlled release with first order kinetics over 9 days.

An overall reduction in post-implantation impedance for microelectrode sites that were coated with the dexamethasone-eluting material was shown. An average reduction of $25 \%$ in impedance at the Peak Resistance Frequency was observed in comparison to control devices, demonstrating that the tissue reaction was reduced and the drug-eluting material was effective.

\section{References}

[1] K.D. Wise, Silicon microsystems for neuroscience and neural prostheses, IEEE Engineering in Medicine and Biology Magazine 24 (2005) 22-29.

[2] A.B. Schwartz, Cortical neural prosthetics, Annual Review of Neuroscience 27 (2004) 487-507.

[3] D.H. Szarowski, et al., Brain responses to micro-machined silicon devices, Brain Research 983 (2003) 23-35.

[4] J.N. Turner, et al., Cerebral astrocyte response to micromachined silicon implants, Experimental Neurology 156 (1999) 33-49.

[5] A. Mercanzini, et al., In vivo electrical impedance spectroscopy of tissue reaction to microelectrode arrays, Biomedical Engineering IEEE Transactions on 56 (2009) 1909-1918.

[6] J.C. Williams, et al., Long-term neural recording characteristics of wire microelectrode arrays implanted in cerebral cortex, Brain Research Protocols 4 (1999) 303-313.

[7] E. Azemi, et al., Surface immobilization of neural adhesion molecule L1 for improving the biocompatibility of chronic neural probes: in vitro characterization, Acta Biomaterialia 4 (2008) 1208-1217.

[8] Y.H. Zhong, R.V. Bellamkonda, Controlled release of anti-inflammatory agent alpha-MSH from neural implants, Journal of Controlled Release 106 (2005) 309-318.

[9] Y. Kato, et al., Preliminary Study of Multichannel Flexible Neural Probes Coated with Hybrid Biodegradable Polymer, Presented at the Annual International Conference of the IEEE Engineering in Medicine and Biology, 2006.

[10] L. Spataro, et al., Dexamethasone treatment reduces astroglia responses to inserted neuroprosthetic devices in rat neocortex, Experimental Neurology 194 (2005) 289-300.

[11] Y. Zhong, R.V. Bellamkonda, Dexamethasone-coated neural probes elicit attenuated inflammatory response and neuronal loss compared to uncoated neural probes, Brain Research 1148 (2007) 15-27.

[12] D.H. Kim, D.C. Martin, Sustained release of dexamethasone from hydrophilic matrices using PLGA nanoparticles for neural drug delivery, Biomaterials 27 (2006) 3031-3037.

[13] W. Franks, et al., Impedance characterization and modeling of electrodes for biomedical applications, IEEE Transactions on Biomedical Engineering 52 (2005) 1295-1302. 
[14] V.S. Polikov, et al., Response of brain tissue to chronically implanted neural electrodes, Journal of Neuroscience Methods 148 (2005) 1-18.

[15] X. Liu, et al., Stability of the interface between neural tissue and chronically implanted intracortical microelectrodes, IEEE Transactions on Rehabilitation Engineering 7 (1999) 315-326.

[16] R.J. Vetter, et al., Chronic neural recording using silicon-substrate microelectrode arrays implanted in cerebral cortex, IEEE Transactions on Biomedical Engineering 51 (2004) 896-904.

[17] S. Takeuchi, et al., Parylene flexible neural probes integrated with microfluidic channels, Lab on a Chip 5 (2005) 519-523.

[18] P.J. Rousche, et al., Flexible polyimide-based intracortical electrode arrays with bioactive capability, IEEE Transactions on Biomedical Engineering 48 (2001) 361-370.

[19] S. Metz, et al., Flexible polyimide probes with microelectrodes and embedded microfluidic channels for simultaneous drug delivery and multi-channel monitoring of bioelectric activity, Biosensors \& Bioelectronics 19 (2004) 1309-1318.

[20] A. Mercanzini, et al., Demonstration of cortical recording using novel flexible polymer neural probes, Sensors and Actuators A: Physical 143 (2008) 90-96.

[21] K.C. Cheung, et al., Flexible polyimide microelectrode array for in vivo recordings and current source density analysis, Biosensors \& Bioelectronics 22 (2007) 1783-1790.

[22] T. Moon, et al., Buckling strength of coated and uncoated silicon microelectrodes, Annual International Conference of the IEEE Engineering in Medicine and Biology Proceedings, vol. 2, 2003, pp. 1944-1947.

[23] S.T. Reddy, et al., In vivo targeting of dendritic cells in lymph nodes with poly (propylene sulfide) nanoparticles, Journal of Controlled Release 112 (2006) 26-34.

[24] A. Rehor, et al., Novel carriers based on polysulfide nanoparticles: production via living emulsion polymerization, characterization and preliminary carrier assessment, Journal of Controlled Release vol. 87 (2003) 246-247 Feb 21.
[25] S.T. Reddy, et al., Targeting dendritic cells with biomaterials: developing the next generation of vaccines, Trends in Immunology 27 (2006) 573-579.

[26] S.T. Reddy, et al., Exploiting lymphatic transport and complement activation in nanoparticle vaccines, Nature Biotechnology 25 (2007) 1159-1164.

[27] A. Rehor, et al., Oxidation-sensitive polymeric nanoparticles, Langmuir 21 (2005) 411-417.

[28] G. Kwon, et al., Block copolymer micelles for drug delivery: loading and release of doxorubicin, Journal of Controlled Release 48 (1997) 195-201.

[29] G. Paxinos, C. Watson, The Rat Brain in Stereotaxic Coordinates, 5th edElsevier Academic Press, Amsterdam; Boston, 2005.

[30] W. Shain, et al., Controlling cellular reactive responses around neural prosthetic devices using peripheral and local intervention strategies, IEEE Transactions on Neural Systems and Rehabilitation Engineering 11 (2003) 186-188.

[31] W.M. Saltzman, M.L. Radomsky, Drugs released from polymers - diffusion and elimination in brain-tissue, Chemical Engineering Science 46 (1991) 2429-2444.

[32] A. Mercanzini, et al., The effect of Biodegradable Drug Release Coatings on the Electrical Characteristics of Neural Electrodes, Transducers and Eurosensors '07 4th International Conference on Solid-State Sensors, Actuators and Microsystems, 2007, pp. 1377-1380.

[33] J.C. Williams, et al., Complex impedance spectroscopy for monitoring tissue responses to inserted neural implants, Journal of Neural Engineering 4 (2007) 410-423.

[34] W. He, et al., Nanoscale laminin coating modulates cortical scarring response around implanted silicon microelectrode arrays, Journal of Neural Engineering 3 (2006) 316-326.

[35] W. He, R.V. Bellamkonda, Nanoscale neuro-integrative coatings for neural implants, Biomaterials 26 (2005) 2983-2990.

[36] P.J. Rousche, et al., Flexible polyimide-based intracortical electrode arrays with bioactive capability, IEEE Transactions on Biomedical Engineering 48 (2001) 361-371. 